\section{Cureus}

Received 03/06/2013

Review began 03/08/2013

Review ended 03/31/2014

Published 04/01/2014

C) Copyright 2014

Alizadeh et al. This is an open access article distributed under the terms of the Creative Commons Attribution License CC-BY 3.0., which permits unrestricted use, distribution, and reproduction in any medium, provided the original author and source are credited.

\title{
Abdominal Adiposity and Testosterone Levels in Patients with Localized Prostate Cancer
}

Moein Alizadeh ${ }^{1}$, Nhu Tram Nguyen ${ }^{2}$, Marie-Pierre Sylvestre ${ }^{3}$, Thu-van Nguyen ${ }^{4}$, JeanPierre Guay ${ }^{4}$, Thomas Zilli ${ }^{5}$, Jean-Paul Bahary ${ }^{4}$, Daniel Taussky ${ }^{4}$

1. Centre Hospitalier de l'Université de Montréal, Centre Hospitalier de L'Université de Montréal 2. Department of Radiation Oncology, Juravinski Cancer Centre-McMaster University 3. Department of biostatistics, Centre de recherche du CHUM, Centre hospitalier de l'Université de Montréal (CHUM) 4. Department of Radiation Oncology, Centre hospitalier de l'Université de Montréal (CHUM) 5. Department of Radiation Oncology, Hôpitaux Universitaires de Genève (HUG)

$\square$ Corresponding author: Moein Alizadeh, moein.alizadeh@gmail.com Disclosures can be found in Additional Information at the end of the article

\section{Abstract}

Objectives: To analyze the relationship between body mass index (BMI), fat tissue distribution, and total testosterone (TT) levels in men with localized prostate cancer (PCa).

Methods: Two hundred and thirty-eight patients with either intermediate-risk PCa (60.5\%) or high-risk PCa (39.5\%) treated by external beam radiotherapy (EBRT), with or without androgen deprivation therapy (ADT), were analyzed. The visceral adipose tissue (VAT) and subcutaneous adipose tissue (SAT) were identified from patient's radiotherapy planning computed tomography (CT) images. Patients were divided into two groups: the first included patients whose TT levels were hypogonadal; the second included patients whose TT levels were normal.

Results: There was a correlation between VAT and BMI ( $\mathrm{r}=0.627)$. In an univariate model, BMI $(\mathrm{p}=0.0174)$ and VAT $(\mathrm{p}=0.0228)$ were both predictive for low TT; age $(\mathrm{p}=0.579)$ was not. In a multivariate analysis taking into account the non-linear relationship between a low TT and BMI, BMI was the only predictive factor of a low TT ( $\mathrm{p}=0.0304)$. An increase in BMI from 15 to $35 \mathrm{~kg} / \mathrm{m} 2$ was associated with a fairly linear increase in the probability of having a low TT level; an increase in BMI of $5 \mathrm{~kg} / \mathrm{m} 2$ resulted in an increase in probability of a low TT of $10 \%$. On the other hand, a BMI $>35 \mathrm{~kg} / \mathrm{m} 2$ had a lower probability of a low TT level.

Conclusions: There is a non-linear relationship between hypogonadal TT levels and BMI. VAT was not an independent predictor for low TT levels. Our results emphasize the important relationship between low TT and obesity in patients with PCa.

Categories: Radiation Oncology, Urology

Keywords: obesity, prostate cancer, testosterone, bmi

\section{Introduction}

Hypogonadism and visceral adiposity are linked and often coexist in the same subjects, together with insulin resistance and metabolic syndrome (MetS) [1], but most data are from cross-sectional studies and do not allow to make a causal inference [2]. Not only do obesity and total testosterone (TT) seem to share a common link, they both have a similar influence on 
prostate cancer. It was recently hypothesized that low TT levels in obese patients may predispose men to more poorly-differentiated cancer, which might explain the higher mortality rate for prostate cancer in obese men [2]. Some studies showed a relationship between low TT levels and PCa, low TT and higher Gleason score (GS), higher tumour burden, higher rate of bilaterality, and decreasing TT levels as risk of progression raised, and in metastatic disease, with poorer response to androgen deprivation therapy (ADT) and shorter overall survival [3-5].

Although the literature is inconclusive regarding the influence of obesity on prostate cancer, obesity has been associated with an increased risk for future diagnosis of high-risk prostate cancer [6, 8-9]: obesity either protects, promotes, or has no effect at all [7]. One reason for these conflicting results could be that there were different measures of adiposity used, such as body mass index (BMI) and hip-to-waist ratio [7], both of which are not direct measures of visceral adipose tissue (VAT). Men have a predominantly visceral fat accumulation [8]. Body fat distribution is regulated by sex hormones. A higher ratio of VAT is associated with many health disorders, including many metabolic disorders [8]. VAT can be determined by computed tomography (CT) or magnetic resonance imaging (MRI) [9]. A recent study found that visceral obesity was significantly higher in patients with prostate cancer than in controls [10]. Since both low TT and visceral obesity share certain links and exert a similar influence on prostate cancer aggressiveness, we investigated the relationship between low TT and visceral obesity in patients presenting with localized prostate cancer.

\section{Materials And Methods}

\section{Patient characteristics and treatment}

Between March 2003 and September 2007, 144 patients with intermediate-risk prostate cancer were enrolled in a prospective multicenter phase III randomized trial comparing two different dose levels of external beam radiotherapy (EBRT). Included were patients with clinical stage T1b-2b (American Joint Commission on Cancer, 6th Edition), a GS of 7, prostate-specific antigen (PSA) levels of $<15 \mathrm{ng} / \mathrm{mL}$, or GS scores of $\leqslant 6$ and PSA $\geqslant 10$ but $\leqslant 20 \mathrm{ng} / \mathrm{mL}$.

Another 94 patients were randomized to a phase III multicenter randomized study for patients with high-risk disease, defined by at least one of the following three risk factors: clinical stage T3 or T4, GS 8-10, or PSA > 20 ng/mL. Patients received 70 Gy. Randomization occurred between 18 and 36 months of neoadjuvant and adjuvant ADT. Both studies were approved by the Human Ethics Committee of the Centre Hospitalier de l'Université de Montréal (ethics identification numbers ND 02.042 and ND 01.011).

\section{Anthropometric measures of obesity}

BMI (defined as weight in kilograms divided by height in meters squared $[\mathrm{kg} / \mathrm{m} 2]$ ) was categorized according to the World Health Organization's definition (normal weight, BMI < 25 $\mathrm{kg} / \mathrm{m} 2$; overweight, 25-29.9 kg/m2; obesity, $\geqslant 30 \mathrm{~kg} / \mathrm{m} 2$ ). Patients of Asian descent were excluded (other BMI cut-off applicable). Weight and height were measured and recorded at the first clinical visit.

VAT and subcutaneous adipose tissue (SAT) were measured at the iliac crest level, since this often corresponds with the fourth lumbar vertebra. VAT and SAT were delineated with the EclipseTM (Varian Medical System, Palo Alto, USA) treatment planning system on three 3-mm slices. VAT/SAT (\%) ratio was calculated for statistical analysis.

\section{Clinical follow-up and statistical analysis}

Patients were followed every three months for the first two years, every six months for an 
additional three years, and then annually thereafter. TT was measured at baseline within four weeks after registration by automated immunoassay systems but before start of EBRT and ADT. Blood samples were analyzed by different laboratories. As a result, different reference values were applied.

\section{Statistical analysis}

The first goal was to find factors associated with low TT levels. To that aim, patients were divided into two groups: the first group consisted of patients whose TT levels were below the normal reference values (low TT) at baseline before start of treatment, the second group consisted of patients whose TT levels were above the lowest reference value (normal levels). The range of TT in patients who had a value below normal at baseline was $1-9 \mathrm{nmol} / \mathrm{L}$ (mean 6.78 , standard deviation [SD] 2.5), and for patients with a normal value, it was 7-29 nmol/L (mean 13.4, SD 4.4) ( $\mathrm{P}<0.001$ for difference in TT between both groups). Clinical characteristics between both groups were compared using a chi-square test or non-parametric Mann-Whitney $\mathrm{U}$ test if the distribution of the variable did not allow for the use of a parametric test.

Univariate and multivariate logistic models were used to estimate the association between obesity and low TT. Two multivariate models were considered and compared using Akaike's Information Criterion: Model 1 included BMI, age, and VAT, while Model 2 included VAT/SAT, age, and VAT. Linearity of effect was assessed by testing quadratic term for each of the independent variables.

In the second part of the statistical analysis, patients who did not receive any hormonal treatment (patients in the intermediate risk group only), who had a normal TT before treatment and whose subsequent TT results were under the reference value were investigated to explore which factors were potentially associated with that change.

Statistical analysis was done using R (R Foundation for Statistical Computing, Vienna, Austria).

\section{Results}

Two hundred and forty-three patients were available for evaluation. Five patients (2.1\%) had missing values and were therefore excluded from analysis. One hundred and forty-four patients (60.5\%) were treated for intermediate-risk prostate cancer and 94 (39.5\%) for high-risk cancer. In the intermediate group, $77.1 \%$ of patients had a normal TT, and $67 \%$ of patients in the high risk group had a normal TT at baseline. Table 1 lists patient characteristics: patients with low TT levels did not have more aggressive disease than patients with a normal level before EBRT. 


\section{Cureus}

\begin{tabular}{|c|c|c|c|c|}
\hline Characteristics & All & Normal TT & Low TT & $\mathbf{P}^{\mathbf{a}}$ \\
\hline \multicolumn{5}{|l|}{ PSA (ng/mL) } \\
\hline Continuous & - & - & - & 0.845 \\
\hline$<20$ & 81.5 (194) & 59.7 (142) & 21.8 (52) & 0.950 \\
\hline$\geq 20$ & $18.5(44)$ & 13.5 (32) & $5.0(12)$ & - \\
\hline \multicolumn{5}{|l|}{ Gleason score } \\
\hline$\leq 6$ & $16.8(40)$ & $10.9(26)$ & $5.9(14)$ & 0.241 \\
\hline$\geq 7$ & 83.2 (198) & $62.2(148)$ & $21.0(50)$ & - \\
\hline \multicolumn{5}{|l|}{ Cilnical I-stage } \\
\hline T1-T2 & 80.3 (191) & $60.1(143)$ & $20.2(48)$ & 0.270 \\
\hline T3 & $19.7(47)$ & $13.0(31)$ & $6.7(16)$ & - \\
\hline \multicolumn{5}{|l|}{ Risk classes } \\
\hline Intermediate-risk & 60.5 (144) & 45.4 (108) & $15.1(36)$ & 0.102 \\
\hline High-risk & 39.5 (94) & 27.7 (66) & $11.8(28)$ & - \\
\hline \multicolumn{5}{|l|}{ Age (y) } \\
\hline$<70.8$ & 49.2 (117) & 35.7 (85) & 13.4 (32) & 0.884 \\
\hline$\geq 70.8$ & $50.8(121)$ & $37.4(89)$ & $13.4(32)$ & - \\
\hline
\end{tabular}

\section{TABLE 1: Patient characteristics stratified by total testosterone (TT ) $(n=238)$. Values}

are in percentage (n)

${ }^{a}$ Chi-square test b Mann-Whitney U test

The median BMI was $27.6 \mathrm{~kg} / \mathrm{m} 2$ (range: $15.5-51.5 \mathrm{~kg} / \mathrm{m} 2$ ). Fifty-seven patients (23.9\%) had a normal BMI, 110 (46.2\%) were overweight (BMI 25-29.9 kg/m2), and 71 (29.8\%) were obese (BMI $>30 \mathrm{~kg} / \mathrm{m} 2$, including $8.8 \%>35 \mathrm{~kg} / \mathrm{m} 2, \mathrm{n}=21$ ). There was a correlation between visceral fat and BMI $(r=0.627, p=<0.0001)$. Age showed only a weak correlation with BMI $(r=-0.292, p<0.0001)$ and VAT/SAT $(\mathrm{r}=0.204, \mathrm{p}=0.002)$.

The difference in mean BMI between patients with normal compared to low TT was $1.3 \mathrm{~kg} / \mathrm{m} 2$ $(\mathrm{p}=0.005)$. Patients with normal TT before RT had significantly less VAT $(\mathrm{P}=0.020)$. Surprisingly, patients with a normal TT were not younger than patients with a low TT ( $\mathrm{p}=0.632$, Table 2$)$. 


\section{Cureus}

\begin{tabular}{|c|c|c|c|c|}
\hline & OR & $95 \% \mathrm{Cl}$ & P-Value & *Joint Test P-Value \\
\hline Age & 0.9904 & $0.9395-1.0453$ & 0.1230 & \\
\hline BMI & 1.6632 & $1.0642-2.8383$ & 0.0396 & 0.0304 \\
\hline BMI squared & 0.9930 & $0.9847-0.9999$ & 0.0677 & \\
\hline VAT/SAT & 2.1237 & $0.7149-6.2949$ & 0.1719 & \\
\hline
\end{tabular}

\section{TABLE 2: Multivariate analysis for factors having an influence on low total testosterone level.}

${ }^{*} \mathrm{p}$-value for the joint effect of BMI modelled as BMI + BMI squared.

In an univariate model, BMI $(\mathrm{p}=0.0174)$ and VAT $(\mathrm{p}=0.0228)$ were both predictive of a low TT, whereas VAT/SAT $(0.1068)$ and age $(\mathrm{p}=0.579)$ were not.

In a logistic linear model, including age and VAT/SAT, BMI had a better predictive value (Akaike's Information Criterion, AIC $=276.85$ ) than the model with visceral fat (AIC = 278.18). In the multivariate model with visceral fat, none of the factors was predictive of a low TT, not even VAT $(p=0.108)$

The association between a low TT and BMI was not linear and was thus modelled by a linear and a quadratic term. The effect of BMI on the probability of low TT, with $95 \%$ point-wise confidence interval, is shown in Figure 1. This figure indicates that an increase in BMI from 15 to $35 \mathrm{~kg} / \mathrm{m} 2$ is associated with a fairly linear increase in the probability of having a low TT level but that probability decreases for larger BMI. For BMI below $35 \mathrm{~kg} / \mathrm{m} 2$, an increase in BMI of 5 $\mathrm{kg} / \mathrm{m} 2$ is associated with an increase of probability of a low TT of roughly $10 \%$. The confidence bands are wide for large BMI; therefore, these results have to be interpreted with caution.

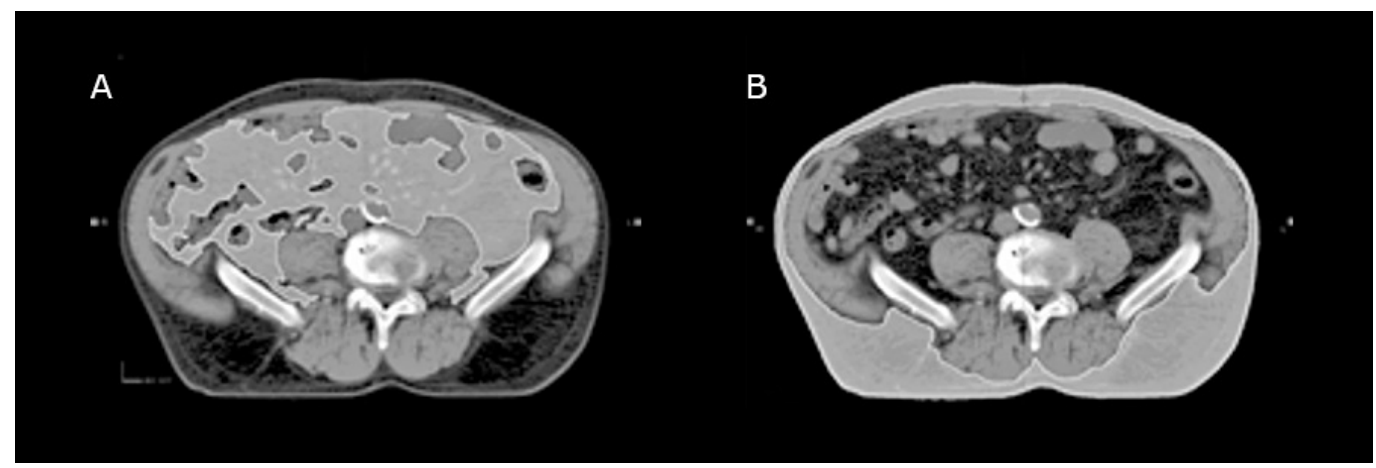

FIGURE 1: Abdominal adiposity as measured on a computed tomography image.

A. Visceral adipose tissue B. Subcutaneous adipose tissue 
were compared for 142 patients in the intermediate risk group at baseline and after a median follow-up of 37.6 months. A total of 33 (23.2\%) patients had a normal TT and subsequently a low TT-level. In this sample, none of the factors was a significant predictor of a subsequent low TT: BMI $(p=681)$, VAT $(p=0.895)$, VAT/SV $(p=0.712)$, and age $(p=0.592)$. None of the nonlinearity checks gave rise to a better fit.

\section{Discussion}

The diagnosis of hypogonadism requires the presence of low serum TT levels and the presence of hypogonadal symptoms [11]. Hypogonadism can affect general health and quality of life by decreasing sex drive and performance, decreasing muscle mass and strength, decreased bone mineral density, and increasing body fat, particularly abdominal fat [12]. Adipose tissue is an active endocrine and metabolic organ that produces numerous adipokines, vascular endothelial growth factor (VEGF), tumour necrosis factor-alpha (TNF- $\alpha$ ) and interleukin-6 (IL-6) [13]. The association between hypogonadism and obesity is complex. There is an intricate relationship between sex hormones, adiposity and body fat distribution; TT levels are reduced in abdominal obesity [8, 14-15]. Visceral obesity can probably be considered a relevant cause of hypogonadism, but at the same time, hypogonadism could be a cause of obesity, establishing a vicious cycle $[2,16]$.

A hypothesis for the association between TT and obesity is that high estrogen levels generated by the aromatization of TT in adipose tissue suppress testicular TT production [17-18]. In addition, it has been hypothesized that androgen deficiency plays a central role in the process of adipogenesis and increased VAT [16]. Travison, et al. [19] reported in a prospective cohort study that men who became obese (BMI $\geqslant 30 \mathrm{~kg} / \mathrm{m} 2)$ experienced a decline in TT comparable to that associated with 10 years of aging.

To our knowledge, this is the first study to investigate in intermediate and high risk PCa, TT and abdominal fat distribution measured by CT. Measuring visceral fat by CT is more accurate than BMI because BMI does not account for muscle mass which varies with age. Nevertheless, we found that BMI is superior to direct VAT measurements in predicting for low TT in patients with localized prostate cancer. Interestingly, it seems that TT levels seem to increase in very obese patients, yet the $95 \%$ confidence intervals are very large and this must be confirmed in a separate study.

Our data contradicts Tsai, et al. (20) who reported a significant but not strong $(r=-0.26, \mathrm{P}=$ 0.006) inverse relationship between TT levels and intra-abdominal fat measured by abdominal CT in Japanese-American men. To our surprise, age did not have an influence on TT levels. The lack of association between low TT levels and age is probably due to the fact that there was relatively little variance of age in our patients (median $70.8 \mathrm{y}$, SD 6 y). Others have shown that TT levels decrease with age: the prevalence of hypogonadal levels in the Baltimore Longitudinal Study of Aging [21] was 20\% for men in their sixties and 30\% for men in their seventies.

An expert roundtable discussion about testosterone measurements in prostate cancer [4] concluded that current evidence linking more aggressive prostate cancer with low TT is equivocal. In their review on the relationship between obesity and prostate cancer, Freedland and Platz [7], state that it is possible that lower free testosterone in obese men predispose them to more aggressive prostate cancer. For patients in this study, low TT levels was not associated to GS, PSA or clinical T-stage. Because of the short follow-up, we did not analyze the influence of low TT levels on treatment outcomes and we can not exclude that on follow-up, patients with hypogonadal levels will respond unfavourably to treatment. However, we do not anticipate this, since others have recently shown that TT does not influence biochemical failure after EBRT [22] or brachytherapy [23]. 
Our study is a retrospective study and has its limitations. We did not have data on waist circumference or waist-to-hip ratio. Most studies analyzed the relationship between TT and BMI and waist-to-hip ratio as a marker for central abdominal obesity [15]. Some could argue that only one sample of TT at a given time yet is insufficient to confirm a hypogonadal TT level. But previous studies have also relied on one unique measurement [24]. Yet, we refrained from categorising our patients as hypogonadal nonetheless. Another shortfall of our study can be that TT was not taken at a precise time, given the circadian variability of TT. Yet, it has been shown that the circadian variation is blunted or non-significant in elderly men, which comprises the vast majority of our patients [25-26].

\section{Conclusions}

In conclusion, we found that low TT levels at diagnosis of prostate cancer and on follow-up after primary radiotherapy depended not on age, but on a patient's BMI and not on intraabdominal fat distribution. A relatively small reduction in BMI could potentially lead to normal TT levels and better quality of life.

\section{Additional Information \\ Disclosures}

Human subjects: All authors have confirmed that this study did not involve human participants or tissue. Animal subjects: All authors have confirmed that this study did not involve animal subjects or tissue. Conflicts of interest: In compliance with the ICMJE uniform disclosure form, all authors declare the following: Payment/services info: All authors have declared that no financial support was received from any organization for the submitted work. Financial relationships: All authors have declared that they have no financial relationships at present or within the previous three years with any organizations that might have an interest in the submitted work. Other relationships: All authors have declared that there are no other relationships or activities that could appear to have influenced the submitted work.

\section{References}

1. Bruce KD, Byrne CD: The metabolic syndrome: common origins of a multifactorial disorder . Postgrad Med J.. 2009, 85:614-621. 10.1136/pgmj.2008.078014

2. Corona G, Mannucci E, Forti G, Maggi M: Hypogonadism, ED, metabolic syndrome and obesity: a pathological link supporting cardiovascular diseases. Int J Androl.. 2009, 32:587598. 10.1111/j.1365-2605.2008.00951

3. Severi G, Morris HA, MacInnis RJ, et al.: Circulating steroid hormones and the risk of prostate cancer. Cancer Epidemiol Biomarkers Prev.. 2006, 15:86-91. 10.1158/1055-9965.EPI-05-0633

4. Schulman CC, Irani J, Morote J, et al.: Testosterone measurement in patients with prostate cancer. Eur Urol.. 2010, 58:65-74. 10.1016/j.eururo.2010.04.001

5. Platz EA, Leitzmann MF, Rifai N, et al.: Sex steroid hormones and the androgen receptor gene CAG repeat and subsequent risk of prostate cancer in the prostate-specific antigen era. Cancer Epidemiol Biomarkers Prev.. 2005, 14:1262-1269. 10.1158/1055-9965.EPI-04-0371

6. Wright ME, Chang SC, Schatzkin A, et al.: Prospective study of adiposity and weight change in relation to prostate cancer incidence and mortality. Cancer. 2007, 109:675-684. 10.1002/cncr.22443

7. Freedland SJ, Platz EA: Obesity and prostate cancer: making sense out of apparently conflicting data. Epidemiol Rev. . 2007, 29:88-97. 10.1093/epirev/mxm006

8. Matsuzawa Y, Shimomura I, Nakamura T, Keno Y, Kotani K, Tokunaga K: Pathophysiology and pathogenesis of visceral fat obesity. Obes Res.. 1995, 3:187-194.

9. Wajchenberg BL, Giannella-Neto D, da Silva ME, Santos RF: Depot-specific hormonal characteristics of subcutaneous and visceral adipose tissue and their relation to the metabolic syndrome. Horm Metab Res.. 2002, 34:616-621. 10.1055/s-2002-38256

10. von Hafe P, Pina F, Perez A, Tavares M, Barros H: Visceral fat accumulation as a risk factor for 
prostate cancer. Obes Res.. 2004, 12:1930-1935. 10.1038/oby.2004.242

11. Dandona P, Rosenberg MT.: A practical guide to male hypogonadism in the primary care setting. Int J Clin Pract.. 2010, 64:682-696. 10.1111/j.1742-1241.2010.02355.x

12. Miner M, Canty DJ, Shabsigh R: Testosterone replacement therapy in hypogonadal men: assessing benefits, risks, and best practices. Postgrad Med.. 2008, 120:130-153. 10.3810/pgm.2008.09.1914

13. Stroup SP, Cullen J, Auge BK, L'Esperance JO, Kang SK: Effect of obesity on prostate-specific antigen recurrence after radiation therapy for localized prostate cancer as measured by the 2006 Radiation Therapy Oncology Group-American Society for Therapeutic Radiation and Oncology (RTOG-ASTRO) Phoenix consensus definition. Cancer. 2007, 110:1003-1009. 10.1002/cncr.22873

14. Kley HK, Deselaers T, Peerenboom H: Evidence for hypogonadism in massively obese males due to decreased free testosterone. Horm Metab Res. 1981, 13:639-641. 10.1055/s-20071019359

15. Tan RS, Pu SJ: Impact of obesity on hypogonadism in the andropause . Int J Androl. 2002, 25:195-201. 10.1046/j.1365-2605.2002.00356.x

16. Traish AM, Guay A, Feeley R, Saad F: The dark side of testosterone deficiency: I. Metabolic syndrome and erectile dysfunction. J Androl. 2009, 30:10-22. 10.2164/jandrol.108.005215

17. Cohen PG: The hypogonadal-obesity cycle: role of aromatase in modulating the testosteroneestradiol shunt--a major factor in the genesis of morbid obesity. Med Hypotheses. 1999, 52:49-51. 10.1054/mehy.1997.0624

18. Kapoor D, Malkin CJ, Channer KS, Jones TH: Androgens, insulin resistance and vascular disease in men. Clin Endocrinol (Oxf). 2005, 63:239-250. 10.1111/j.1365-2265.2005.02299.x

19. Travison TG, Araujo AB, Kupelian V, O'Donnell AB, McKinlay JB: The relative contributions of aging, health, and lifestyle factors to serum testosterone decline in men. J Clin Endocrinol Metab. 2007, 92:549-555. 10.1210/jc.2006-1859

20. Tsai EC, Boyko EJ, Leonetti DL, Fujimoto WY: Low serum testosterone level as a predictor of increased visceral fat in Japanese-American men. Int J Obes Relat Metab Disord. 2000, 24:485491.

21. Harman SM, Metter EJ, Tobin JD, Pearson J, Blackman MR: Longitudinal effects of aging on serum total and free testosterone levels in healthy men. J Clin Endocrinol Metab. 2001, 86:724-731. 10.1210/jc.86.2.724

22. Roach M, 3rd, Bae K, Lawton C, et al: Baseline Serum Testosterone in Men Treated with Androgen Deprivation Therapy and Radiotherapy for Localized Prostate Cancer. Int J Radiat Oncol Biol Phys. 2010, 78:1314-1322. 10.1016/j.ijrobp.2009.09.073

23. Taira AV, Merrick GS, Galbreath RW, et al: Pretreatment serum testosterone and androgen deprivation: effect on disease recurrence and overall survival in prostate cancer patients treated with brachytherapy. Int J Radiat Oncol Biol Phys. 2009, 74:1143-1149.

10.1016/j.ijrobp.2008.09.046

24. Basaria S, Coviello AD, Travison TG, et al: Adverse events associated with testosterone administration. N Engl J Med. 2010, 363:109-122. 10.1056/NEJMoa1000485

25. Plymate SR, Tenover JS, Bremner WJ: Circadian variation in testosterone, sex hormonebinding globulin, and calculated non-sex hormone-binding globulin bound testosterone in healthy young and elderly men. J Androl. 1989, 10:366-371.

26. Bremner WJ, Vitiello MV, Prinz PN: Loss of circadian rhythmicity in blood testosterone levels with aging in normal men. J Clin Endocrinol Metab. 1983, 56:1278-1281. 